\title{
DOPAMINA E 0 RIM NA SEPSE: UMA REVISÃO SISTEMÁTICA
}

\author{
Denise Varella Katz*, eduardo Juan Troster, flavio Adolfo Costa Vaz \\ Estudo realizado na Unidade de Terapia Intensiva do Instituto da Criança do Hospital das Clínicas \\ da Faculdade de Medicina da Universidade de São Paulo, São Paulo, SP.
}

RESUMO - A insuficiência renal aguda (IRA) tem uma alta morbimortalidade em pacientes de terapia intensiva. A sepse grave e choque séptico são fatores de risco importantes para o desenvolvimento de IRA. A dopamina em dose baixa $(0,5 \mathrm{a} 3 \mu \mathrm{g} / \mathrm{kg} / \mathrm{min})$ vem sendo empregada durante várias décadas como opção terapêutica para a proteção da função renal nestes pacientes, ainda que na ausência de estudos bem controlados.

Oвјетіvos. Verificar evidências na literatura que justifiquem o uso rotineiro da dopamina em dose baixa nos pacientes com sepse grave e choque séptico.

Métodos. Busca sistemática da literatura, abrangendo bancos de dados eletrônicos (MEDLINE, EMBASE, LILACS) e a busca manual de artigos.

Resultados. Dos cinco estudos clínicos randomizados encontra- dos, nenhum se enquadrou nos critérios de inclusão, pois não avaliavam a função renal. Dentre os estudos do tipo séries de casos, apenas oito foram passíveis de avaliação qualitativa. Foram descritos alguns efeitos adversos associados à dopamina, tais como aumento do shunt pulmonar, taquiarritmias, aumento na pressão de artéria pulmonar, que não foram estatisticamente significantes. A mortalidade também não se alterou com o uso da dopamina.

ConclusöEs. Não existem evidências suficientes na literatura que suportem o uso rotineiro da dopamina em dose baixa como opção terapêutica para proteção da função renal na sepse grave e choque séptico.

Unitermos: Dopamina. Rim. Sepse. Choque séptico.

\section{INTRODUÇÃO}

A insuficiência renal aguda (IRA) é uma sindrome clínica caracterizada por queda rápida na filtração glomerular, alteração na distribuição extravascular de fluidos, distúrbios na homeostase de eletrólitos e equilíbrio ácidobase, e na retenção de nitrogênio proveniente do catabolismo protéico'.

Em sua grande maioria, a IRA intrínseca é induzida por isquemia e/ou nefrotoxinas, e classicamente está associada com necrose das células epiteliais do túbulo renal (NTA) $)^{1,2,3}$.

Nos pacientes internados em terapia intensiva, a grande maioria das agressões renais têm como fisiopatogenia a lesão direta ou a lesão isquêmica. Assim, a disfunção renal nestes pacientes abrange os mecanismos prérenal e renal.

A IRA ocorre em aproximadamente $5 \%$ das admissões hospitalares e em até $30 \%$ das admissões em terapia intensiva. A oligúria ocorre em $50 \%$ dos casos. Apesar de reversível em grande parte dos casos, a IRA está associada comaltas taxas de morbi-mortalidade, geralmente relacionando-se a graves doenças de base e sérias complicações ${ }^{2,4,5}$.

* Correspondência:

R. Presidente Antonio Cândido, 350 Ap. 82 05083-060 - São Paulo - SP
A necrose tubular aguda (NTA) difere da insuficiência renal pré-renal porque está associada à lesão do parênquima renal, não se resolvendo imediatamente após a restauração da perfusão. A NTA é o resultado de hipoperfusão mais intensa e prolongada, e geralmente está associada a outras afecções como sepse e nefrotoxinas, além de pacientes submetidos a grandes cirurgias, trauma, e grandes queimaduras. A sepse induz à hipoperfusão renal devido à ocorrência de vasodilatação sistêmica e vasoconstricção renal. Ainda, a endotoxina sensibiliza o tecido renal aos efeitos deletérios da isquemia!.

O diagnóstico da insuficiência renal aguda baseia-se principalmente na história sugestiva de lesão renal e em dados laboratoriais, podendo ou não haver sinais clínicos de insuficiência renal ${ }^{1,2}$. Clinicamente, a IRA pode se manifestar pela presença de edema, hipertensão, insuficiência cardíaca congestiva, alterações decorrentes da uremia, tais como confusão mental, coma, sangramentos etc. Está acompanhada de oligúria em aproximadamente $50 \%$ dos casos: diurese menor que $400 \mathrm{ml} /$ dia ou menor que $0,5 \mathrm{ml} / \mathrm{kg} / \mathrm{hora}$ em adolescentes e adultos, e menor que $1 \mathrm{ml} / \mathrm{kg} /$ hora em lactentes e crianças'. Do ponto de vista laboratorial, o diagnóstico de IRA baseia-se na análise seriada de uréia e creatinina séricas, porém esses índices têm baixa sensibilidade para a medida da função glomerular.

A incidência de sepse em crianças apresenta um padrão bimodal, com o primeiro pico no período neonatal, e o segundo por volta de dois anos. Dentre todas as admissões em UTI pediátrica, a incidência de sepse chega a $24 \%$, com mortalidade de $10 \%$ a $50 \%$.

De acordo com Bone e et al. ${ }^{59}$, foram propostas as definiçõos para sepse, sepse grave e choque séptico; adaptadas por Hayden $(1994)^{6}$ para a faixa etária pediátrica(Tabela I).

Sepse é a resposta sistêmica à infecção, que inclui duas ou mais das seguintes manifestações, na presença de infecção:

a) temperatura corpórea $>38^{\circ} \mathrm{C}$ ou $<36^{\circ} \mathrm{C}$ b) frequência cardíaca $>90$ batimentos $/ \mathrm{min}$ c) taquipnéia ( $F R>20 / \mathrm{min}$ ) ou hiperventilação ( $\mathrm{pCO}_{2}<32$ torr)

d) contagem de leucócitos $>12.000 / \mathrm{mm}^{3}$ ou $<4.000 \mathrm{~mm}^{3}$, ou presença de mais de $10 \%$ de bastonetes.

Sepse grave é definida como a sepse associada à disfunção orgânica, alteração relacionada à hipoperfusão, ou hipotensão. Alterações relacionadas à hipoperfusão incluem acidose lática, oligúria ou alteração aguda do nível de consciência. 
Tabela I - Terminologia para sepse (ACCP/SCCM) - adaptada para crianças por $\mathrm{Hayden}^{6}$

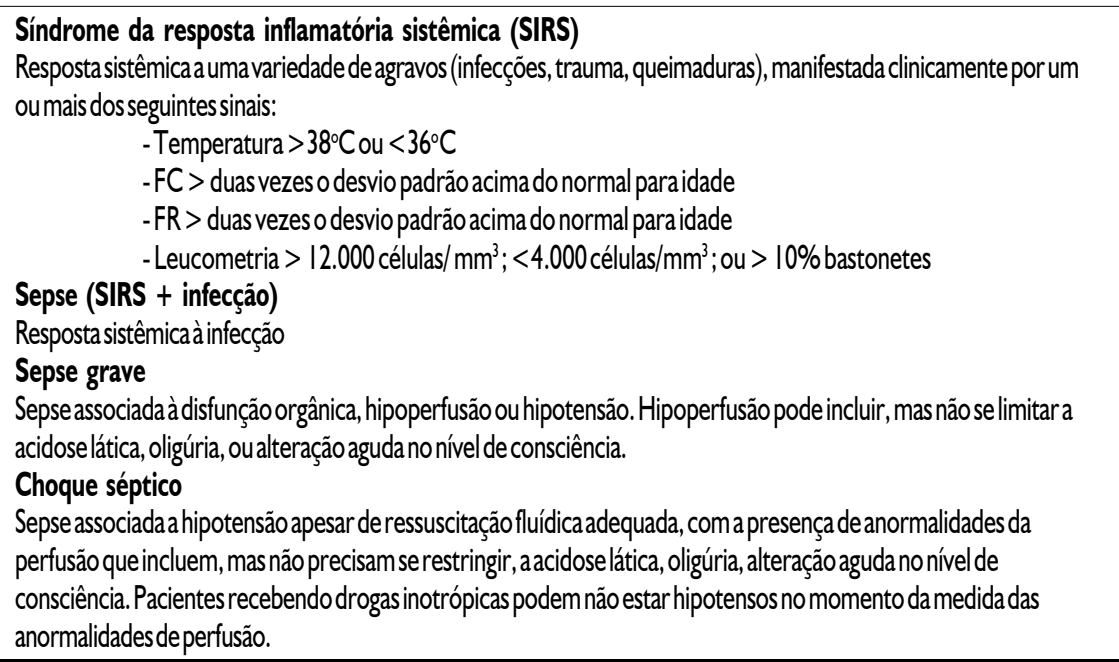

Choque séptico é a sequência da sepse grave, sendo definido como hipotensão induzida pela sepse, que é persistente apesar de ressuscitação fluídica adequada, e se acompanha de anormalidades relacionadas à hipoperfusão ou disfunção orgânica (acidose lática, oligúria, alteração do nível de consciência). Pacientes recebendo drogas inotrópicas podem não estar hipotensos no momento da medida das anormalidades de perfusão.

Em terapia intensiva, vários esquemas são propostos com o objetivo de evitar ou minimizar a lesão renal com a instalação de IRA, dentre eles podendo-se citar o emprego de diuréticos, de bloqueadores de canais de cálcio e da dopamina. A administração de manitol antes da agressão isquêmica tem demonstrado algum grau de proteção renal apenas em modelos experimentais. Os estudos clínicos do manitol na IRA são em sua maioria não-controlados. $O$ manitol pode reverter a oligúria em alguns pacientes se administrado numa fase precoce da IRA, porém sem melhorar a função renal ${ }^{8}$. Os bloqueadores de canais de cálcio têm se mostrado eficazes em vários estudos experimentais de modelos isquêmicos e vasoconstrictores de IRA; porém, ensaios clínicos não apresentam tais resultados, havendo inclusive a possibilidade de ocorrerem efeitos hemodinâmicos negativos nestes pacientes?.
A dopamina teria espaço em pacientes oligúricos pelo seu efeito de ativar os receptores adrenérgicos renais aumentando o FPR, a TFG e a excreção de sódio, quando administrada em baixas doses $(0,5 \mathrm{a} 2 \mu \mathrm{g} / \mathrm{kg} /$ $\mathrm{min})$. Vários estudos confirmam o efeito da dopamina de induzir a diurese, mas não esclarecem a dúvida quanto à sua capacidade de prevenir a IRA na sepse grave e choque séptico. Assim como com os diuréticos, o aumento do débito urinário pode ou não contribuir para uma maior sobrevida9,10.

Adopamina foi inicialmente descrita paraa utilização clínica na década de 60 , e vem sendo empregada desde então nos pacientes hemodinamicamente instáveis, em todas as faixas etárias, com o objetivo de proteger a função renal quando administrada em doses dopaminérgicas.

Pouco se conseguiu comprovar no que diz respeito ao efeito da dopamina em baixas doses sobre a circulação esplâncnica. Em estudos com animais e com voluntários humanos saudáveis, a infusão de dopamina levou a um aumento do clearance de inulina, do fluxo plasmático renal e da excreção de sódio ${ }^{53}$. Mousdale et al. ${ }^{54}$ demonstrou em humanos saudáveis que a dopamina nas doses de 2,5; 5,0 e $10,0 \mu \mathrm{g} / \mathrm{kg} / \mathrm{min}$ induziu a um aumento significantemente maior no fluxo plasmático renal quando comparada à dobu- tamina em doses similares e à dopexamina nas doses de I,0;2,0 e $4,0 \mu \mathrm{g} / \mathrm{kg} / \mathrm{min}$. Leier et al. ${ }^{55}$ demonstrou em pacientes com ICC que a infusão de dopamina $3 \mu \mathrm{g} /$ $\mathrm{kg} /$ min aumentou significantemente ofluxo sanguíneo renal e não alterou o fluxo sanguíneo esplâncnico.

\section{Objetivos}

Verificar o potencial efeito protetor renal da dopamina em dose dopaminérgica, em pacientes com sepse grave e choque séptico

Avaliar o desfecho clínico com relação a: a) diferenças na mortalidade entre os grupos estudados (com e sem dopamina);

b) ocorrência de efeitos adversos relacionados ao uso da dopamina.

\section{Metodologia}

\section{Técnica empregada}

Revisão sistemática da literatura, que consiste na aplicação de estratégias científicas que limitem vieses para a reunião sistemática, apreciação crítica e síntese de todos os estudos relevantes no período estudado. Quando possível, realizou-se a metanálise, ou seja, a revisão sistemática quantitativa que emprega métodos estatísticos para combinar e sumariar os resultados de vários estudos.

\section{Critérios de seleção}

a) Tipos dos estudos selecionados:

Para a revisão sistemática qualitativa:

- Ensaios clínicos controlados e randomizados

-Estudos de "coorte"

-Estudos do tipo caso-controle

- Descrição de séries de casos

Para a metanálise, incluimos apenas os ensaios clínicos controlados e randomizados.

\section{Seleção dos pacientes}

b) Foram incluídos:

- lactentes, crianças e adolescentes, na faixa etária de I mês a 17 anos, internados em terapia intensiva (UTI) com diagnóstico de sepse grave ou choque séptico, e que se apresentassem no início do estudo com a função renal normal.

- adultos internados em UTI, com diagnóstico de sepse grave ou choque séptico, e que se apresentavam no início do estudo com a função renal normal. 


\section{Intervenção}

Utilização de dopamina em dose dopaminérgica $(0,5$ a $3 \mu \mathrm{g} / \mathrm{kg} / \mathrm{min})$ comparada a controles submetidos a tratamento sem a dopamina.

d) Desfechos avaliados

- variação do débito urinário (considerandose oligúria menos que I ml/kg/hora de diurese)

- variação do nível sérico de creatinina

- variação no clearance de creatinina

- complicações relacionadas ao uso da dopamina

- morte durante o estudo

e) Estratégia de busca para a identificação dos estudos

A revisão bibliográfica foi realizada de forma manual e informatizada, sendo consultados os seguintes bancos de dados: MEDLINE (período de 1966 a 1998), LILACS (período de 1980 a 1998) e EMBASE (período de 1980 a 1998), e usadas as palavras-chave na busca informatizada (substituídas pelos MeSH terms - "medical subject heading" - DESC): dopamine, kidney, sepsis, septic shock, child, adolescence.

f) Critérios para a avaliação metodológica dos estudos

Os estudos foram avaliados do ponto de vista de qualidade metodológica de acordo com os critérios de Heyland et al. ", apresentados na Tabela 2. Quanto maior o rigor metodológico do estudo, maior a sua pontuação, sendo 13 o escore máximo.

\section{g) Métodos estatísticos}

Os dados foram avaliados através do programa REVIEW MANAGER, versão 3.I, The Cochrane Collaboration, março de 1998; os intervalos de confiança foram definidos em $95 \%$, e a homogeneidade dos estudos foi avaliada pelo programa REVIEWMANAGER, versão 3.I, The Cochrane Collaboration, março de 1998, pelo teste do qui-quadrado. O erro tipo I adotado (erro $\alpha$ ) foi de 0,05 .

\section{Resultados}

\section{Busca sistemática das publicações}

Foramidentificados um total de 124 estudos. O primeiro autor a estudar o efeito renal da dopamina, numa série de casos, foi Goldberg et al. $(1963)^{12}$, em pacientes com insuficiência cardíaca congestiva.

\begin{tabular}{|c|c|c|c|}
\hline \multicolumn{4}{|c|}{ Tabela 2 - Critérios de qualidade metodológica (HEYLAND et al., 1996)" } \\
\hline & \multicolumn{3}{|c|}{ ESCORE } \\
\hline & 0 & 1 & 2 \\
\hline MÉTODOS & $\overline{\text { Não randomizado }}$ & - & $\begin{array}{l}\text { Verdadeiramente } \\
\text { randomizado }\end{array}$ \\
\hline Randomização & & & \\
\hline Mascaramento & nãomascarado & duplo-cegamento & - \\
\hline Análise & Outra & - & intençãodetratar \\
\hline POPULAÇÃO & & & \\
\hline Seleçãode pacientes & $\begin{array}{l}\text { pacientesselecionados, } \\
\text { ousem especificação } \\
\text { clara }\end{array}$ & $\begin{array}{l}\text { pacientes eleitos } \\
\text { consecutivamente }\end{array}$ & - \\
\hline $\begin{array}{l}\text { Comparabilidade dos } \\
\text { grupos no ińicio do estudo }\end{array}$ & $\begin{array}{l}\text { Não comparáveis, ou } \\
\text { nãoseguramente } \\
\text { comparáveis }\end{array}$ & Comparáveis & - \\
\hline $\begin{array}{l}\text { Extensão do seguimento } \\
\text { INTERVENCÃO }\end{array}$ & $<100 \%$ & $100 \%$ & - \\
\hline Protocolodetratamento & Maldescrito & Reprodutível & - \\
\hline $\begin{array}{l}\text { Co-intervenções } \\
\text { todasiguais }\end{array}$ & Não descritas & Descritas, mas não iguais & Bem descritase \\
\hline "Crossovers" & Nãodescritos & $\begin{array}{l}\text { ounão bemespecificadas } \\
>10 \%\end{array}$ & $<10 \%$ \\
\hline
\end{tabular}

Tabela 3 - Distribuição dos estudos encontrados na revisão sistemática

\begin{tabular}{lcccc}
\hline & 1971 - 1980 & 1981 - 1990 & 1991 - 2000 & Total \\
$\begin{array}{lccc}\text { Revisöesnão } \\
\text { sistemáticas editoriais }\end{array}$ & 5 & 13 & 40 & 58 \\
Estudosexperimentais & 4 & 10 & 22 & 36 \\
$\begin{array}{l}\text { Caso-controle } \\
\text { Sériesdecasos }\end{array}$ & 5 & 0 & & 0 \\
$\begin{array}{l}\text { Estudoscoorte } \\
\text { Revisöes sistemáticas }\end{array}$ & 0 & 4 & 12 & 21 \\
Estudosclínicos & & 0 & 2 & 2 \\
randomizados & & & 1 & 1 \\
TOTAL & 14 & 1 & 5 & 6 \\
\hline
\end{tabular}

Os estudos encontrados, de acordo coma sua distribuição, estão resumidos na Tabela 3.

\section{Descrição dos estudos}

Aúnica revisãosistemáticaencontrada, Rudis etal. ${ }^{13}$; estudava pacientes com sepse, mas nãofoi incluída no estudo porque não avaliou função renal, e sim a terapêutica dirigida para atingir valores supra-normais de oferta de oxigênio.

Dentre os estudos coorte encontrados, nenhum foi adequado para os desfechos estudados: Brivet et al. ${ }^{14}$ estudaram pacientes já com IRA (critério de exclusão na metodologia), e Liaño et al. ${ }^{15}$ não estudaram a dopamina.
Dentre os cinco ensaios clínicos randomizados encontrados, nenhum avaliou função renal como desfecho, apenas estudaram variáveis hemodinâmicas.

Foram identificados 19 estudos do tipo séries de caso.

Dentre os estudos séries de caso, identificamos para os desfechos procurados um total de I 2 estudos: De La Cal et al. ${ }^{16}$; Desjars et al. ${ }^{17}$; Driscoll et al. ${ }^{18}$; Flancbaum et al. ${ }^{19}$; Girbes et al. ${ }^{20}$; Henderson et al. ${ }^{21}$; Juste et al. ${ }^{22}$; Lherm et al ${ }^{23}$; Rendl-Wenzl et al. ${ }^{24}$; Seriet al. $(1993)^{25}$; Seri et al. $(1998)^{26}$ e VélizPintos et al. ${ }^{27}$. 
Seri $1993^{25}$ e Seri $1998^{26}$ foram excluídos por utilizarem recém-nascidos como população estudada. O estudo de Driscoll et al. ${ }^{18}$ foi excluído por avaliar a função renal apenas utilizando o desfecho "diurese". Finalmente, o estudo de Girbes et al. ${ }^{20}$ também foi excluído por ter sido publicado de forma incompleta. Assim, restou-nos um total de oito estudos do tipo séries de casos para a avaliação qualitativa do grupo de pacientes com sepse grave e choque séptico.

De acordo com a faixa etária da população estudada, foram identificados quatro estudos utilizando crianças, dois com recém-nascidos, e 20 estudos com adultos. Dos que utilizaram crianças, apenas Véliz-Pintos et al. ${ }^{27}$ foi incluído na avaliação qualitativa para sepse grave $\mathrm{e}$ choque séptico.

Avaliação qualitativa para dopamina e função renal em pacientes com sepse grave e choque séptico

Foi realizada uma avaliação qualitativa de oito estudos do tipo séries de casos, pois não foi possível a metanálise dos ensaios clínicos randomizados.

De la Cal et al., $1984{ }^{16}$, estudaram sete adultos com choque séptico, utilizando como critérios diagnósticos uma queda na pressão arterial (PA), oligúria e acidose metabólica. Administraram dopamina para todos os pacientes, numa dose que variou de 3 até $27 \mu g /$ $\mathrm{kg} / \mathrm{min}$. Dentre os desfechos avaliados, os autores mediram diurese, clearance de inulina, clearance de paraminohipurato, fração de filtração e fração de excreção de sódio. Observou-se em todos os pacientes um aumento estatisticamente significante da diurese, e em apenas seis casos houve aumento na fração de filtração renal (estatisticamente não significante). Os demais parâmetros não sofreram alterações. Não houveram efeitos colaterais relacionados ao uso da dopamina.

Desjars et al. $\left(1989^{17}\right)$ descreveram 25 adultos com choque séptico (definido como PA sistólica menor ou igual a $90 \mathrm{mmHg}$; oligúria; febre e leucocitose; bacteremia ou identificação de um sítio de infecção). Todos os pacientes receberam volume e norepinefrina para restabelecer a PA, e medidas hemodinâmicas e de função renal foram obtidas antes da administração de norepinefrina e após 24 horas, com a adição de dopamina em dose dopaminérgica. Nos resultados, obser- vou-se um aumento da diurese em todos os pacientes, sendo significantemente maior durante a infusão de epinefrina. A média de creatinina plasmática reduziu, e o clearance de creatinina aumentou de forma significativa durante a administração de norepinefrina.

Flancbaum etal. $\left(1994^{19}\right)$ descreveram 19 adultos em terapia intensiva cirúrgica, alguns deles com diagnóstico de sepse grave, após ressuscitação hídrica adequada. A infusão de dopamina na dose de $2,8 \pm 0,37 \mu \mathrm{g} / \mathrm{kg} / \mathrm{min}$ resultou somente em aumento da diurese de forma estatisticamente significante.

Henderson et al. (19802I), estudando II adultos em terapia intensiva (cinco deles com sepse), concluíram que a introdução de dopamina I $\mu \mathrm{g} / \mathrm{kg} / \mathrm{min}$ em pacientes com tendência à oligúria levou somente a um aumento estatisticamente significante na diurese e na excreção de sódio.

Juste et al. (199822) avaliaram I7 adultos com choque séptico pelos critérios diagnósticos de Bone, já recebendo norepinefrina. Foi administrada a dopamina $2,5 \mu \mathrm{g} / \mathrm{kg} / \mathrm{min}$ a todos os pacientes em dois períodos consecutivos de 2 horas, com um intervalo de 4 horas sem a droga, e tomadas as medidas ao final de cada período. Dentre os resultados estatisticamente significantes, descreveu-se uma queda no volume urinário e na fração de excreção de sódio quando se descontinuou a dopamina, sem ter havido aumento nesses valores quando a droga foi reintroduzida. $O$ clearance de creatinina não se alterou.

Lherm et al. $\left(1996^{23}\right)$ avaliaram dois grupos de adultos, um com sepse grave (grupol; $n=14$ ) e outro com choque séptico (grupo 2; $n=15$ ), segundo os critérios diagnósticos de Bone. Ambos os grupos receberam dopamina $2 \mu \mathrm{g} / \mathrm{kg} / \mathrm{min}$. No grupo I observou-se aumento da diurese num período de 48 horas, aumento da excreção de sódio e do clearance de creatinina, todos estatisticamente significantes. No grupo 2 não se observaram alterações.

Rendl-Wenzl etal. $\left(1993^{24}\right)$, estudando 56 adultos com choque séptico refratário a dopamina em doses de até $20 \mu \mathrm{g} / \mathrm{kg} / \mathrm{min}$, dividiram em dois grupos. $\mathrm{O}$ grupo I, com pacientes normotensos, recebeu dopamina 2,5 $\mu \mathrm{g} / \mathrm{kg} / \mathrm{min}$ e dobutamina. $\mathrm{O}$ grupo 2 , com pacientes hipotensos, recebeu norepinefrina em doses variáveis (até elevar a PA) e dobutamina. Dentre as medidas para avaliação da função renal, observou-se aumento do clearance de creatinina após 24 e 48 horas. Não houve alteração no débito urinário e clearance de água livre. Cinco de sete pacientes que desenvolveram IRA não oligúrica recuperaram a função renal.

Véliz-Pintos et al. (199427) estudaram 35 crianças com choque séptico com o objetivo de avaliar desfecho e complicações clínicas. Seus critérios diagnósticos não seguiram a normatização preconizada por Bone ${ }^{59}$. Em várias fases de seu estudo, este autor administrou albumina, metilprednisolona, naloxone, dobutamina e dopamina na dose de $5 \mu \mathrm{g} / \mathrm{kg} /$ $\min \mu \mathrm{g} / \mathrm{kg} / \mathrm{min}$. Ele descreve a insuficiência renal aguda como complicação presente em $30 \%$ dos casos, sem no entanto descrever os critérios para o diagnóstico de IRA.

Dentre todos os estudos, é difícil de se realizar uma avaliação crítica, pois as amostras de pacientes são na maioria das vezes heterogêneas, os critérios diagnósticos para sepse, choque séptico e IRA são diversos ou até não declarados, e os desfechos avaliados são os mais variados. A dopamina nem sempre é a variável estudada, havendo outros fatores de confusão para o desfecho.

\section{Mortalidade e efeitos adversos}

Os efeitos adversos eamortalidade relacionadosà dopamina, descritos em todos os estudos clínicos, estão resumidos nas Tabelas 4 até 7.

\section{Discussão}

\section{Revisão sistemática}

Os passos a serem tomados na prática da Medicina Baseada em Evidências (EBM) são simples e claros:

I) identificar uma informação necessária e formular claramente uma questão clínica em termos de população, intervenção (ou evento), e o(s) desfecho(s) procurado(s);

2) buscar a evidência relevante, em estudos primários em humanos ou em fontes de evidência sintetizada;

3) analisar criticamente a evidência encontrada; 4) aplicar a evidência à prática clínica.

Quando se pretende estudar um medicamento, como a dopamina, existem algumas questões a serem feitas sobre a significância clínica do tratamento ${ }^{44}$ :

- Qual a magnitude do risco basal da doença (IRA)? Ou seja, sem o tratamento, que proporção de pacientes terá má evolução? 


\begin{tabular}{|c|c|c|c|c|c|}
\hline Marik etal. $1994^{28}$ & $\begin{array}{l}\text { Pacientes } \\
\text { adultos } 20\end{array}$ & $\begin{array}{l}\text { Tipo } \\
\text { sepse }\end{array}$ & $\begin{array}{l}\text { Mortalidade } \\
60 \% \text { grupo } \\
\text { dopaminaX50\%grupo } \\
\text { norepinefr }\end{array}$ & $\begin{array}{l}\text { Efeito Adv. } \\
\text { NR }\end{array}$ & $\begin{array}{l}\text { Nota } \\
\text { Não avaliafunção renal }\end{array}$ \\
\hline Martin etal. $1993^{29}$ & adultos 32 & sepse & $1 / 16$ & $\mathrm{NR}$ & Nãoavaliafunçãorenal \\
\hline Hayes et al. $1997^{30}$ & adultos 78 & sepse & NR & NR & Não avaliafunção renal, nem dopamina \\
\hline Gilbertetal. $1986^{31}$ & adultos 54 & sepse & $\begin{array}{l}6 \% \text { em } 24 \mathrm{~h} \\
65 \% \text { tardia }\end{array}$ & NR & Não estuda dopamina \\
\hline Hanneman et al. $1995^{32}$ & adultos 25 & sepse & $\begin{array}{l}7 / 15 \text { grupo Dobutamina } \\
8 / 10 \text { grupo Dobuta }+ \\
\text { Noradrenalina }\end{array}$ & $\begin{array}{l}\text { Aum PoAP; } \\
\text { aum shunt pulmonar } \\
\text { aumFC; queda } \mathrm{PaO2} \\
\text { quedaRVS }\end{array}$ & Nãoestuda dopamina em dose baixa \\
\hline
\end{tabular}

NR:não relatado; IAM: infarto agudo de miocárdio;PoAP: pressão de oclusão de artéria pulmonar; RVS: resistência vascular sistêmica

\begin{tabular}{|c|c|c|c|c|c|}
\hline & Pacientes & Tipo & Mortalidade & Efeit & Nota \\
\hline Liaño et al. $1998^{15}$ & adultos críticos 253 & $\mathbb{R A}$ & $\begin{array}{l}\text { UTI }=71,5 \% \\
\text { outros }=31,5 \%\end{array}$ & NR & $\begin{array}{l}\text { Nãoavalia } \\
\text { dopamina } \\
\text { nemfunção } \\
\text { renal }\end{array}$ \\
\hline Brivetetal. $1996^{14}$ & adultos com IRA 360 & IRA & $58 \%$ & NR & $\begin{array}{l}\text { Não estuda } \\
\text { dopamina }\end{array}$ \\
\hline
\end{tabular}

\begin{tabular}{|c|c|c|c|c|c|}
\hline & Pacientes & Tipo & Mortalidade & Efeit & Nota \\
\hline Rudis et al. $1996^{13}$ & MEDLINE & sepse & NR & NR & $\begin{array}{l}\text { Nãoavalia } \\
\text { função renal }\end{array}$ \\
\hline
\end{tabular}

- O tratamento é eficaz? Ou, existe um efeito real e não devido ao acaso?

- Quais são as direções e magnitudes do efeito terapêutico?

- Há efeitos indesejáveis atribuíveis ao tratamento com a dopamina?

- Quais são os custos econômicos e as conseqüências do tratamento?

- Os benefícios clínicos do tratamento compensam os efeitos indesejáveis e os custos econômicos?

- A quais pacientes esses resultados são aplicáveis?

I) Formulando a questão: uma boa revisão sistemática baseia-se numa questão bem formulada: "A dopamina tem ou não efeito protetor renal nos pacientes com sepse grave e choque séptico?". A questão dirige o estudo no momento em que define quais os estudos que serão incluídos, qual seráa estratégia de busca paraidentificar os estudos primariamente relevantes, e quais os dados que deverão ser extraídos de cada estudo. Formular uma questão pobre resultaria numa revisão pobre.

2) Tipos de estudos incluídos: a revisão deve sempre se basear nas melhores evidências disponíveis; ou seja, os estudos a serem incluídos na revisão devem utilizar métodos que eliminem ao máximo os vieses ao responderem a questão formulada. Assim, as revisões sistemáticas para tratamento e prevenção devem incluir estudos randomizados, controlados, preferencialmente aqueles que utilizem um método cego de alocação. Revisões sistemáticas para métodos diagnósticos devem incluir estudos que comparem independentemente um ou mais testes com um método bem estabelecido (padrão ouro). Antigamen- te, revisões questionando fatores de risco deviam incluir estudos relevantes caso-controle, coorte, idealmente com análises multivariadas para ajustar a outros fatores de risco bem estabelecidos. Quando não se encontram estudos com o melhor nível de evidência, pode ser apropriado considerar outros níveis de evidência. Assim, para estudarmos a dopamina, buscamos preferencialmente os estudos clínicos randomizados (que não obedeciam os critérios de inclusão); e estudos do tipo séries de caso, caracterizando um nível de qualidade metodológica imediatamente inferior. Outros detalhes particularmente considerados foram a necessidade de cegamento nos ECR e a presença de fatores de confusão.

3) Critérios de inclusão: quão abrangentes devem ser os critérios de inclusão? Além da questão ser clinicamente relevante, os critérios de inclusão devem ser suficientemente sensíveis. Se alguma característica dos pacientes em qualquer grupo pode alterar significantementeo desfecho, então essas características devem ser levadas em conta. Entretanto, critérios de inclusão estritos demais podem limitar os dados na revisão, aumentando assim o risco de se obterem resultados falso-positivos ou falso-negativos. Em nosso estudo, devido ao baixo número de estudos encontrados, tivemos que agrupar estudos com adultos e crianças, e estudos só com adultos ou só com crianças.

4) Estratégias de Busca: o banco de dados eletrônico (MEDLINE, EMBASE) permite acesso relativamente rápido a grandes quantidades de artigos publicados. Porém, estudos têm mostrado que, dependendo do tópico, uma pesquisa MEDLINE identifica apenas de 


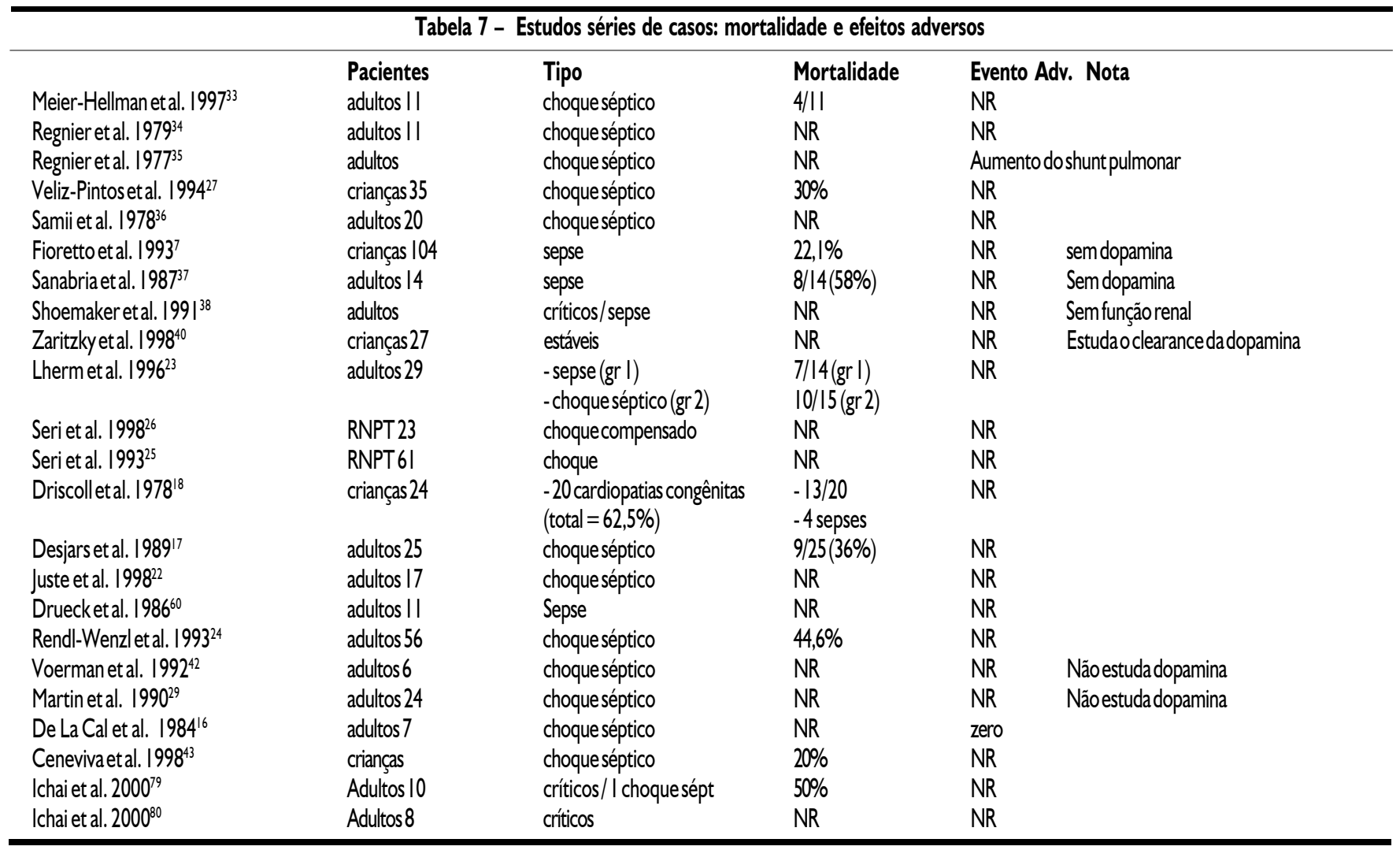

$32 \%$ a $91 \%$ dos estudos controlados randomizados publicados em revistas que estão indexadas ao MEDLINE. Isto se dá em parte porque a indexação é inadequada, ou porque foram utilizados termos incorretos na estratégia de busca. Mesmo com uma ótima estratégia, a busca eletrônica não identifica artigos de revistas que não estão indexadas no banco de dados. Para minimizar este problema, vários bancos de dados devem ser acessados. Em nosso estudo, procedemos a busca informatizada (MEDLINE, LILACS, EMBASE), utilizando termos adequados, além da busca manual em revistas e em bibliografias de artigos, e o contato direto com pesquisadores, o que tornou o nosso levantamento suficientemente completo ${ }^{45}$.

Vieses na localização e seleção dos estudos:

- Estudos com resultados significantes têm maior probabilidade de serem publicados do que estudos sem resultados significantes, levando ao viés de publicação.

- Dentre os estudos publicados, aqueles com resultados significantes são mais freqüentemente publicados em inglês, mais freqüen- temente citados, e mais repetidamente publicados, levando a viés de língua, viés de citação, e viés de publicação múltipla.

-Em países menos desenvolvidos, estudos com resultados significantes têm maior probabilidade de serem publicados em periódicos indexados em bancos de dados eletrônicos, 0 que pode levar ao viés de banco de dados.

- Os critérios para a inclusão de estudos em uma metanálise podem ser influenciados pelo conhecimento dos resultados de estudos potentes, levando ao viés de inclusão $0^{46}$.

O valor da metanálise "falha": em alguns casos, uma metanálise conclusiva pode se tornar impossível, tentando-se manter os princípios metodológicos. Nessas "metanálises falhas", os métodos de tratamento, tratamentos simultâneos, tempo de seguimento, características dos participantes do estudo, ou objetivos finais que foram medidos podem se mostrar variados demais para permitir uma combinação sensível dos resultados. Uma metanálise exclusivamente baseada em um pequeno número de estudos freqüentemente será inconclusiva, mesmo que a estimativa calculada do efeito seja significante ${ }^{47}$.
Em nosso estudo, podemos considerar que todos esses fatores citados consistiram em obstáculos para a obtenção de resultados positivos. Tratamentos simultâneos com outras catecolaminas em pacientes hemodinamicamente instáveis podem, através de efeito inotrópico, melhorar a pressão de perfusão renal, com ou sem a utilização da dopamina em baixas doses. Estudos que incluíram pacientes adultos e crianças, ou pacientes com diversas patologias, não podem obter conclusões que sejam generalizáveis para qualquer tipo de doente. Por fim, estudos que objetivaram principalmente as medidas hemodinâmicas, quase nunca documentaram de forma homogênea e adequada os parâmetros de função renal.

No presente estudo, a avaliação de sua qualidade nos permite afirmar ${ }^{49}$.

I. Os artigos selecionados tinham como objetivo as questões formuladas por esta revisão sistemática.

2. A busca de estudos em três bancos de dados informatizados e mais a busca manual visou não perder nenhum estudo relevante sobre 0 assunto. 
3. Os critérios de seleção de artigos foram rigorosos, obedecendo à risca os critérios de exclusão.

4. Avalidade dos artigos foiverificada por meio de qualidade metodológica.

5. A descrição das etapas da revisão consta na metodologia, para torná-la reprodutível.

6. As diferenças entre os estudos devem ser atribuídas apenas ao acaso. Para a metanálise, devem sempre ser aplicados testes de homogeneidade que demonstraram, com um $p=0,05$, que as eventuais diferenças entre os estudos agrupados deviam-se a causalidade.

7. Os cálculos foram realizados através de um programa de software desenvolvido especialmente para este fim, para que os resultados fossem os mais precisos possíveis.

\section{Dopamina}

Uma das maiores dificuldades em se pesquisar os efeitos da dopamina na função renal é a falha no controle adequado para as suas diversas ações renais diretas e indiretas. Estas incluem: I - aumento do débito cardíaco; 2- aumento da pressão de perfusão renal; 3- efeito "diurético" sobre o túbulo renal; 4-efeito localizado sobrea resistência vascular renal - efeito vasodilatador renal.Émuito difícil diferenciar esses efeitos, especialmente em estudos de investigação clínica, pois ocorre grande interferência entre causas e efeitos.

É muito difícil se relacionar a dose infundida de dopamina com o seu efeito hemodinâmico, provavelmente devido à variabilidade individual no seu clearance. A responsividade vascular periférica às catecolaminas estábastante alterada no choque. Assim, a variabilidade no clearance da dopamina pode adicionar uma instabilidade nessa resposta hemodinâmica. Érecomendado que a infusão de agentes potentes como a dopamina seja titulada para os efeitos hemodinâmicos observados, tais como alterações no débito cardíaco ena resistência vascular sistêmica e pulmonar, frequência cardíaca e pressão arterial, em vez de se basear na idéia de que determinada velocidade de infusão produz um certo efeito padrão $0^{40}$.

Outras catecolaminas que melhoram o débito cardíaco podem também aumentar o FSR. Após cirurgia cardíaca, dobutamina, dopexamina, e dopamina aumentam o FSR proporcionalmente ao aumento do débito cardíaco. Leier et al. ${ }^{55}$ observaram, em pacien- tes com ICC, que a dobutamina produziu um maior aumento no clearance de creatinina do que a dopamina. A adrenalina e a noradrenalina estão sendo cada vez mais utilizadas em pacientes com choque séptico. Em ovelhas com sepse, a adrenalina ( 5 a $40 \mu \mathrm{g} / \mathrm{kg} / \mathrm{min}$ ) produz inicialmente uma queda dose-dependente no FSR, entretanto após 30 minutos ele retoma valores normais e tende a subir. Dados similares têm sido relatados com a noradrenalina ${ }^{58}$.

A dopamina é ou não um vasodilatador renal?

Alguns dados experimentais sugerem que a dopamina pode ter propriedades vasodilatadoras. Vários dos estudos em animais e em humanos foram mal controlados. Inúmeros estudos demonstram um aumento indireto no FSR secundário ao aumento do débito cardíaco, mesmo quando a dopamina é administrada em baixas doses. O efeito "diurético" também é freqüentemente observado, e é atribuído a uma atividade específica da dopamina sobrea célula tubular.

Em termos de proteção renal, as suas propriedades benéficas são:

- aumento na oferta de oxigênio através de um aumento moderado no débito cardíaco, com aumento de FSR;

- potencial diminuição na demanda de oxigênio renal, através da inibição da reabsorção de sódio.

Efeitos deletérios:

- a dopamina prejudica o mecanismo de feedback tubuloglomerular, o que pode afetar adversamente o balanço oferta versus consumo de oxigênio

- a diurese não está sempre associada a um aumento do FSR

- a diurese pode mascarar hipovolemia ou hipoperfusão renal

- uma diurese inapropriada pode gerar hipovolemia.

\section{Avaliação dos estudos clínicos}

Dentre os estudos do tipo séries de caso, a ausência de grupo controle impossibilita qualquer conclusão com relação ao efeito protetor renal da dopamina. Além disso, vários tratamentos simultâneos foram empregados, tais como volume e drogas inotrópicas, fazendo com que a melhora dos parâmetros de função renal não tivesse origem definida (dopamina ou outras medidas terapêuticas?). $O$ número de pacientes em cada estudo foi sempre pequeno ( 7 até 56 ), a maioria deles com doenças de base diversas. Lherm et al. ${ }^{23}$ compararam dois grupos absolutamente diferentes, um com sepse grave e outro com choque séptico, chegando a dois tipos de resultados, um para cada grupo, inferindo assim uma conclusão metodologicamente inadequada.

Todos os estudos, apesar de não fornecerem conclusões para função renal, constataram um aumento no débito urinário quando se utilizou a dopamina em dose baixa, corroborando com este dado já consagrado na literatura, de que a dopamina exerce efeito diurético.

\section{Conclusṍes}

Não foram encontradas evidências científicas suficientes na literatura que justifiquem 0 uso da dopamina como tendo efeito protetor renal em pacientes com sepse grave e choque séptico.

Quanto ao desfecho clínico, temos que:

a) Adiferença encontrada na mortalidade não foi significante. As amostras dos estudos não foram de tamanho adequado (erro $\beta$ grande).

b) Os eventos adversos foram: aumento do shunt pulmonar, taquiarritmias, e aumento da pressão de artéria pulmonar, relatados de forma aleatória, não permitindo uma associação significante destes eventos com o emprego da dopamina.

\section{SUMMARY}

\section{DOPAMINE AND KIDNEY IN SEPSIS: A SYSTEMATIC REVIEW}

Acute renal failure has a high morbidity and mortality in critically ill patients. Severe sepsis and septic shock are important risk factors for the development of acute renal failure. Low-dose dopamine $(0.5$ to $3 \mu \mathrm{g} / \mathrm{kg} / \mathrm{min}$ ) has been used for decades as a renal-protective therapy in such patients, even in the absence of any controlled study to support this concept.

Background. To Check the literature for evidences supporting the routine use of low-dose dopamine in severe sepsis and septic shock.

Methods. Systematic review of the literature, on electronic databasis (MEDLINE, EMBASE and LILACS), and handsearching.

RESULTS. Only five randomized clinical trials were found, but none of them studied renal 
outcomes. Eight cases series studies were included on a qualitative review. Dopamine was associated with some adverse effects, such as increase in pulmonary shunting, tachyarrhythmias, and increase in pulmonary artery pressure, that were not statistically significant. Mortality also did not change with the use of dopamine.

ConCLUSIONS. There are no sufficient evidences in the literature to support the routine use of low-dose dopamine as a renal protective agent in severe sepsis and septic shock. [Rev Assoc Med Bras 2003; 49(3): 317-25]

KeY words: Dopamine. Kidney. Sepsis. Severe sepsis.

\section{REFERÊNCIAS}

I. Brenner BM, editos. The kidney. 5th ed. Philadelphia:W.B. Saunders; 1996.

2. Brady HR, Singer GG. Acute renal failure. Lancet 1995; 346: 1533-40.

3. Nissensson, AR. Acute renal failure: definition and pathogenesis. Kidney Int 1998; 53(Suppl 66):7S-10S.

4. Hou SH, Bushinsky DA, Wish JB, Cohen JJ, Harrington JT. Hospital-acquired renal insufficiency: a prospective study. Am J Med 1983; 74:243-8.

5. Anderson RJ, Linas SL, Berns AS. Nonoliguric acute renal failure. N Engl J Med 1977; 296: II34-8.

6. Hayden WR. Sepsis terminology in pediatrics. J Pediatr 1994; I 24:657-8.

7. Fioretto JR, Moreira FL, Ferrari GF, Bonatto RC, Ricchetti SMQ, Moraes MA. Sepsis em unidade de terapia intensiva pediátrica-UTIP. Rev Paul Pediatria 1993; I I:203-9.

8. Levinsky NG, Bernard DB, Johnston PA. Mannitol and loop diuretics in acute renal failure. In: Brenner BM, editor. Acute renal failure. Philadelphia: W.B. Saunders; 1983. p.7| 2-22.

9. Fischereder M, Trick CKW, Nath K. A Therapeutic strategies in the prevention of acute renal failure. Semin Nephrol 1994; |4:4|-52.

10. Corwin HL. Acute renal failure in sepsis and multiple organ failure. In: Fein AM, editor. Sepsis and multiorgan failure. Maryland: Williams \& Wilkins; 1997. p.546-56.

II. Heyland DK, Cook DJ, King D, Kernerman P, Brun-Buisson P. Maximizing oxygen delivery in critically ill patients: a methodological appraisal of the evidence. Crit Care Med 1996; 24:517-24.

12. Golberg LI, Mcdonald RH, Zimmerman AM. Sodium diuresis produced by dopamine in patients with congestive heart failure. NEngl] Med 1963; 269: 1060-3.

13. Rudis MI, Basha MA, Zarowitz BJ. Is it time to reposition vasopressors and inotropes in sepsis? Crit Care Med 1996; 24:525-37.
14. Brivet FG, Kleinknecht DJ, Loirat $P$, Landais PJM. The French Study Group on Acute Renal Failure. Acute renal failure in intensive care units: causes, outcome, and prognostic factors of hospital mortality: a prospective, multicenter study. Crit Care Med 1996; 24: 192-8.

15. Liaño F, Junco E, Pascual J, Madero R, Verde E. Madrid Acute Renal Failure Study Group. The spectrum of acute renal failure in the intensive care unit compared with that seen in other settings. Kidney Int 1998; 53(Suppl 66):SI6S24. 1998.

16. De La Cal MA, Miravalles E, Pascual T, Esteban A, Ruiz-Santana S. Dose-related hemodynamic and renal effects of dopamine in septic shock. Crit Care Med 1984; 12:22-5.

17. Desjars P, Pinaud M, Bugnon D, Tasseau F. Norepinephrine therapy has no deleterious renal effects in human septic shock. Crit Care Med 1989; 17:426-9.

18. Driscoll DJ, Gillette PC, Mcnamara DG. The use of dopamine in children. J Pediatr 1978; 92:309-14.

19. Flancbaum L, Choban PS, Dasta JF. Quantitative effects of low-dose dopamine on urine output in oliguric surgical intensive care unit patients. Crit Care Med 1994; 22:6I-6.

20. Girbes ARJ, Patten MT, McCloskey BV, Groeneveld AB, Hoogenberg K. The renal and neurohumoral effects of the addition of lowdose dopamine in septic critically ill patients. Intensive Care Med 1996; 26:1685-9.

21. Henderson IS, Beattie TJ, Kennedy AC. Dopamine hydrochloride in oliguric states. Lancet 1980; 2:827-8.

22. Juste RN, Panikkar K, Soni N. The effects of low-dose dopamine infusions on haemodynamic and renal parameters in patients with septic shock requiring treatment with noradrenaline. Intensive Care Med I 998; 24:564-8.

23. Lherm T, Troché G, Rossignol M, Bordes $P$, ZazzoJF. Renal effects of low-dose dopamine in patients with sepsis syndrome or septic shock treated with catecholamines. Intensive Care Med 1996; 22:213-9.

24. Rendl-Wenzl EM, Armbruster C, Edelmann G, Fischl E, Kolacny M, Wechsler-Fördös A, Sporn P. The effects of norepinephrine on hemodynamics and renal function in severe septic shock states. Intensive Care Med 1993; 19:151-4.

25. Seri I, Rudas G, Bors Z, Kanyicska B, Tulassay $T$, Effects of low-dose dopamine infusion on cardiovascular and renal functions, cerebral blood flow, and plasma catecholamine levels in sick preterm neonates. Pediatr Res 1993; 34:742-49.

26. Seri I, Abbasi S, Wood DC, Gerdes JS. Regional hemodynamics effects of dopamine in the sick preterm neonate. J Pediatr 1998; 133:728-34.

27. Véliz-Pintos RA, Pérez-Perafan H, MendozaHernandez M. Evaluación de un algoritmo para el manejo de niños com shock séptico. Rev Mex Pediatria 1994; 61:267-7I.

28. Marik PE, Mohedin M. The contrasting effects of dopamine and norepinefrine on systemic and splancnic oxygen utilization in hyperdynamic sepsis. JAMA 1994; 272: I 354-7.

29. Martin C, Papazian L, Perrin, G, Saux P, Gouin F. Norepinefrine or dopamine for the treatment of hyperdynamic septic shock? Chest 1993; 103:1826-31.

30. Hayes MA, Timmins AC, Yau EHS, Palazzo M, Watson D, Hinds CJ. Oxygen transport patterns in patients with sepsis syndrome or septic shock: Influence of treatment and relationship to outcome. Crit Care Med I 997; 25:926-36.

31. Gilbert EM, Haupt MT, Mandanas RY, Huaringa AJ, Carlson RW. The effect of fluid loading, blood transfusion, and catecholamine infusion on oxygen delivery and consumption in patients with sepsis. Am Rev Respir Dis 1986; 134:873-8.

32. Hannemann L, Reinhart K, Grenzer O, MeierHellmann A, Bredle DL. Comparison of dopamine to dobutamine and norepinefrine for oxygen delivery and uptake in septic shock. Crit Care Med 1995; 23: 1962-69.

33. Meier-Hellmann A, Bredle DL, Specht M, Spies C, Hannemann L, Reinhart K. The effects of low-dose dopamine on splancnic blood flow and oxygen uptake in patients with septic shock. Intensive Care Med 1997; 23:31-7.

34. Regnier B, Safran D, Carlet J, Teisseire B. Comparative haemodynamic effects of dopamine and dobutamine in septic shock. Intensive Care Med 1 979; 5: I I5-20.

35. Regnier B, Rapin M, Gory G, Lemaire F, Teisseire $B$, Harari A. Haemodynamic effects of dopamine in septic shock. Intensive Care Med 1997; 3:47-53.

36. Samii K, Le Gall J-R, Regnier B, Gory G, Rapin $M$. Hemodynamic effects of dopamine in septic shock with and without acute renal failure. Arch Surg 1978; I 13:14 I4-6.

37. Sanabria HR, Hernandez A, Silicani A. Causas de sepsis en la unidad de cuidados intensivos. Diagnostico 1987; 20:165-9.

38. Shoemaker WC, Appel PL, Kram HB. Oxygen transport measurements to evaluate tissue perfusion and titrate therapy: dobutamine and dopamine effects. Critical Care Med 1991; 9:672-88.

39. Eldadah MK, Schwartz PH, Harrisson R, Newth CJL. Pharmacokinetics of dopamine in infants and children. Critical Care Med I99I; 19:1008-II.

40. Zaritsky A, Lotze A, Stull R, Goldstein DS. Steady-state dopamine clearance in critically ill infants and children. Crit Care Med 1988; 16:217-20.

41. Richer M, Robert S, Lebel M. Renal hemodynamics during norepinephrine and low-dose dopamine infusions in man. Crit Care Med 1996; 24: I 1 50-6.

42. Voerman HJ, Stehouwer CDA, Kamp GJ, Schijndel RJ, M, Groeneveld ABJ, Thijs LG. Plasma endotelin levels are increased during septic shock. Crit Care Med 1992; 20:1097-01.

43. Ceneviva G, Paschall JÁ, Maffei F, CarcilloJA. Hemodynamic support in fluid-refractory 
pediatric septic shock. Pediatrics 1998, 102:el9.

44. Sinclair CJ. Systematic reviews of randomized trials. In: Hansen TN, MClintosh N. Current topics in neonatology. Edinbourgh: W.B. Saunders; 1995

45. Counsell C. Formulating questions and locating primary studies for inclusion in systematic reviews. Ann Intern Med 1997; 127:38I-7.

46. Egger M, Smith GD. Meta-analysis: bias in location and selection of studies. BMJ 1998; 316:6I-6.

47. Smith GD, Egger M. Meta-analysis: unresolved issues and future developments. BMJ 1998; 316:221-5.

48. Cassiere HÁ, Groth M, Niederman MS. Evidence-based medicine: the wolf in sheep's clothing. In: Vincent J.-L., editors. Yearbook of intensive care and emergency medicine. New York: Springer; 1998. p.745-9.

49. Cook D. Use of evidence-based medicine in evaluation of sepsis therapies. In: Fein AM, editors. Sepsis and multiorgan failure. Maryland: Williams \& Wilkins; 1997. p.587-95.

50. Fink MP, Baggs AG. Animal models of sepsis and septic shock. In: Fein AM, editor. Sepsis and multiorgan failure. Maryland: Williams \& Wilkins; 1997. p.596-6I3.

5I. Lemeshow S, Teres D, Moseley S. Statistical issues in clinical sepsis trials. In: Fein AM, editor. Sepsis and multiorgan failure. Maryland: Williams \& Wilkins; 1997. p.614-26.

52. Fischer $C$ J, Zheng Y. Interpreting large-scale clinical sepsis trials: a retrospective review. In: Fein AM, editor. Sepsis and multiorgan failure. Maryland:Williams \&Wilkins; 1997. p.627-34.

53. Goldberg LI. Cardiovascular and renal implications of dopamine: potential clinical applications. Pharmacol Rev 1972; 24: I-29.

54. Mousdale S, Clyburn PA, Mackie AM, Groves ND, Rosen M. Comparison of the effects of dopamine, dobutamine and dopexamine upon renal blood flow: a study in normal healthy volunteers. BrJ Clin Pharmacol I 988; 25:555-60.

55. Leier CV. Regional blood flow responses to vasodilators and inotropes in congestive heart failure. Am J Cardiol I 988; 62:86E-93E.
56. Parker S, Carlon GC, Isaacs M, Howland WS, Kahn R. Dopamine administration in oliguria and oliguric renal failure. Critical Care Med 198I; 9:630-2.

57. Polson RJ, Park GR, Lindop MJ. The prevention of renal impairment in patients undergoing orthotopic liver grafting by infusion of low-dose dopamine. Anaesthesia 1987; 42:15-9.

58. Bersten AD, Rutten AJ. Renovascular interaction of epinephrine, dopamine, and intraperitoneal sepsis. Crit Care Med 1995; 23:537-44.

59. American College of Chest Physicians/Society of Critical Care Medicine Consensus Conference: definitions for sepsis and organ failure and guidelines for the use of innovative therapies in sepsis. Crit Care Med 1992; 20:864-74.

60. Drueck C, Welch GW, Pruitt BA. Hemodynamic analysis of septic shock in thermal injury: treatment with dopamine. Am Surg 1978, 44:424-7.

61. Anderson MR, Blummer JL. Advances in the therapy for sepsis in children. Pediatr Clin North Am 1997; 44: 179-205.

62. Aurora RN. Preventing renal failure in critically ill patients [Editorial]. Crit Care Med 1999; 27:2044-5

63. Bellomo R, Cole L, Ronco C. Hemodynamic support and the role of dopamine. Kidney Int 1998; 53(Suppl 66):S7I-S4.

64. Bernard GR. Design and conduct of clinical trials in sepsis and critical illness. In: Fein AM, editor. Sepsis and multiorgan failure. Maryland: Williams \& Wilkins; 1997. p.58I-6.

65. Bernstein PL, Sterns R. Acute renal failure in sepsis and shock. In: Fein AM, editor. Sepsis and multiorgan failure. Maryland: Williams \& Wilkins; 1997. p.243-54.

66. Cook DJ, Sibbald WJ, Vincent JL, Cerra FB. Evidence based medicine: what is it and what can it do for us? Crit Care Med 1996; 24:334-7.

67. Denton MD, Chertow GM, Brady HR. "Renaldose" dopamine for the treatment of acute renal failure: scientific rationale, experimental studies and clinical trials. Kidney Int 1996; 49:4-14
68. Duke GJ, Bersten AD. Dopamine and renal salvage in the critically ill patient. Anaesth Intensive Care 1992; 20:277-302.

69. Kon V, Ichikawa I. Research seminar: physiology of acute renal failure. J Pediatr 1984; 105:351-7.

70. L'abbé KA, Detsky AS, O'rourke K. Metaanalysis in clinical research. Ann Intern Med 1987; 107:224-33.

7I. Lee MR. Dopamine and the kidney. Clin Sci 1982; 62:439-48.

72. Moyer VA, Elliott EJ. Evidence-based pediatrics: the future is now. J Pediatr 2000; 136:5.

73. Naylor CD. Grey zones of clinical practice: some limits to evidence-based medicine. Lancet 1995; 345:840-2.

74. Niederman MS. Evidence based medicine in critical care: Will it take us to the cutting edge ? [Editorial] Crit Care Med 1997; 25: I 448-9.

75. Schwartz LB, Gewertz BL. The renal response to low dose dopamine. J Surg Res 1988; 45:574-88.

76. Szerlip HM. Renal-dose dopamine: fact and fiction. Ann Intern Med I 99 | ; I 5: I 53-4.

77. Thijs A, Thijs LG. Pathogenesis of renal failure in sepsis. Kidney Int 1998; 53(Suppl 66):S34S37.

78. Thijs LG. Kidney protection in sepsis. Acta Anaesthesiol Scand 1998; I:5-8.

79. Ichai C, Soubielle J, Carles M, Giunti C, Grimaud D. Comparison of the renal effects of low to high doses of dopamine and dobutamine in critically ill patients: a singleblind randomized study. Crit Care Med 2000; 28:921-8.

80. Ichai C, Passeron C, Carles M, Bouregba M, Grimaud D. Prolonged low-dose dopamine infusion induces a transient improvement in renal function in hemodynamically stable, critically ill patients: a single-blind, prospective, controlled study. Crit Care Med 2000; 28:1329-35.

Artigo recebido: 03/04/02 Aceito para publicação: 26/02/03 